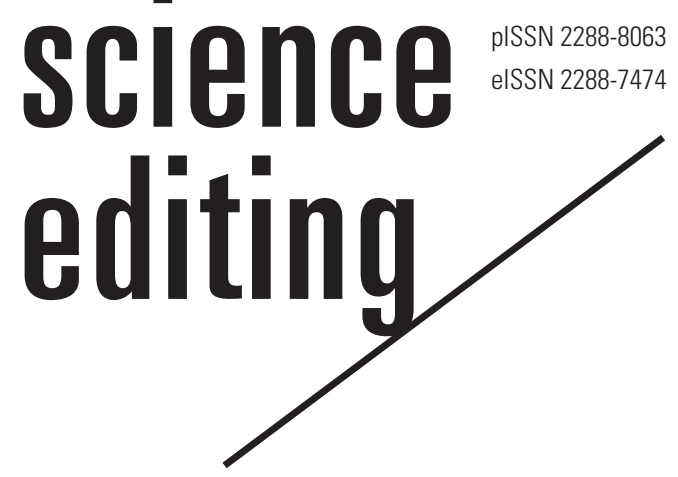

\title{
A bibliometric analysis of COVID-19 research published in nursing journals
}

\author{
Juyeon Oh, Aekyung Kim \\ College of Nursing, Dankook University, Cheonan, Korea
}

Abstract

Purpose: This bibliometric study investigated the current state of documents on coronavirus disease 2019 (COVID-19) published in nursing journals. The analysis focused on the description of most productive journals, institutions, and countries, as well as the characteristics of the documents.

Methods: The publications analyzed in this study were retrieved from the Web of Science database with search keywords. The searches used to establish the study dataset were last updated on July 10, 2020. The inclusion criteria were relevant English-language publications in journals published in the Science Citation Index Expanded or Social Science Citation Index. Content analysis of original articles and reviews was done.

Results: An analysis of 125 publications on COVID-19 from 48 journals showed that the most productive journal and country were the Journal of Clinical Nursing $(\mathrm{n}=18)$ and the USA $(n=53)$, respectively. Original articles $(n=27,21.6 \%)$ and review papers $(n=4,3.2 \%)$ accounted for $24.8 \%$ of the articles, and the highest number of papers were found in early access (published ahead of print) $(n=51,40.8 \%)$ and Q1 journals $(n=73,58.4 \%)$. The content analysis found 10 data-based original articles or reviews, which dealt with the topics of nurse training $(n=2)$, nurses' psychosocial status $(n=2)$, nursing research method$\operatorname{ology}(n=1)$, nursing guidelines $(n=4)$, and protection for nurses $(n=1)$.

Conclusion: This study presents the current situation of nursing research on COVID-19 based on an analysis of publications in nursing journals and provides meaningful information to nursing researchers and editorial board members. The number of data-based original articles on nursing related to COVID-19 remains low.

Received: July 28, 2020

Accepted: August 7, 2020

Correspondence to Aekyung Kim aekim@dankook.ac.kr

ORCID

Juyeon $\mathrm{Oh}$

https://orcid.org/0000-0002-6800-1320 Aekyung Kim

https://orcid.org/0000-0003-1115-6169
Keywords

Bibliography; COVID-19; Scientific journals; Nursing; Web of Science

\section{Introduction}

Background/rationale: The current situation of coronavirus disease 2019 (COVID-19) is unprecedented [1]. Shortly after COVID-19 was identified, it rapidly spread around the world; 
the World Health Organization declared the COVID-19 outbreak as a pandemic on March 11, 2020 [2], and the COVID-19 pandemic has now become a major international concern. In mid-December 2019, the infection was first recognized in Wuhan, Hubei Province, China; as of July 17, 2020, over 13 million confirmed cases of this disease and 580,000 deaths have been reported worldwide [3]. COVID-19 has arguably been the major focus of researchers in relevant fields around the world during the first half of 2020, as shown by the increasing number of publications focused on COVID-19 that have been published since it was first identified [4].

Given the novelty of COVID-19, as well as its global impact, new scientific knowledge provides essential information regarding both the containment of the disease and the management of patients [4]. Scholarly journals are the most important media source for the dissemination of such research findings and information related to connecting this new evidence to practice, as these journals are the most credible and updated information resources for evidence-based practice. In particular, nursing journals play an important role in improving nurses' knowledge of new information and integrating this knowledge into nursing practice. The main target readers of nursing journals are nurses, who are positioned at the very front lines of the battle against COVID-19 [5]. There are approximately 20 million nurses around the world [6], and many of them are currently facing COVID-19 in roles that range from providing bedside nursing care for people affected by the disease to establishing healthcare policies.

Bibliometric analyses provide objective information through the comprehensive assessment of a specific scientific research trend by identifying the number and distribution of publications related to the trend, the publications' authorship and coauthorship, and the most cited articles. Additionally, bibliometric data facilitate both health policy decisions and the allocation of health resources, and they encourage further innovative studies. Several bibliometric analyses on COVID-19 have been published [4,7-10], but there is no bibliometric analysis currently available that specifically focuses on documents published in nursing journals, to the best of our knowledge.

Objectives: The present study aimed to shed light on the current state of the documents published in nursing journals. The analysis focused on describing the most productive journals, institutions, and countries, as well as the characteristics of the relevant documents. As a further analysis, research articles and review papers were evaluated as categories of research.

\section{Methods}

Ethics statement: This research did not involve human subjects, so neither institutional review board approval nor in- formed consent was required.

Study design: This was a descriptive study based on a bibliometric analysis of a literature database.

Data collection: The publications analyzed in this study were retrieved from the Web of Science database (https://clarivate. com/webofsciencegroup), which is maintained by Clarivate Analytics and is one of the most authoritative databases used for such research. The publications were searched using the following search keywords: (TS = COVID-19 OR 2019-nCoV OR coronavirus 2019 OR Coronavirus disease 2019 OR SARS-CoV-2) AND (SU=Nursing). The inclusion criteria consisted of (1) publications in journals published in the Science Citation Index Expanded or Social Science Citation Index and (2) publications written in English. The searches used to establish the study dataset were last updated on July 10 , 2020. This search strategy identified 132 articles, and all the bibliometric details of each article were exported into an Excel spreadsheet ("savedrecs.xls") from the Web of Science website. Seven publications with no information on the author's name or affiliation and the article's digital object identifier were excluded from the analysis. After a review of titles and abstracts by the authors, no publication was excluded for not being relevant to COVID-19. Therefore, the following parameters were extracted from 125 publications and analyzed: authors' information, journal name, volume, issue, document type, reprint address, and date of publication. Additionally, the journals were divided into four groups by their impact factor quartile (Q1, Q2, Q3, and Q4), based on the impact factor ranking from the 2020 Journal Citation Reports. Furthermore, the Web of Science website provides an "analyze report" function, which shows bibliometric information for selected publications, such as the authors' affiliated organization and country/region; we used these data to analyze the most productive countries and institutions.

Statistical methods: The analyses were conducted with Excel 2016 (Microsoft, Redmond, WA, USA) and IBM SPSS Statistics ver. 22.0 (IBM Corp., Armonk, NY, USA). Effects were considered significant when the P-value was less than 0.05 . Descriptive data are presented as numbers, percentages, and rankings. Differences in types of publication and quartile scores depending on the period of publication were analyzed by the chi-square test.

\section{Results}

A total of 125 publications related to COVID-19 were analyzed. Table 1 shows the most productive nursing journals regarding COVID-19. Studies were published in 48 different journals, with the most articles being published in the Journal of Clinical Nursing $(\mathrm{n}=18)$, followed by the Journal of Human 
Table 1. The most productive nursing journals in terms of COVID-19 publications

\begin{tabular}{|c|c|c|c|}
\hline Overall & Number & Original article + review & Number \\
\hline Journal of Human Lactation & 10 & Journal of Clinical Nursing & 3 \\
\hline Heart Lung & 8 & Journal of Human Lactation & 3 \\
\hline Journal of Advanced Nursing & 7 & Bariatric Surgical Practice and Patient Care & 2 \\
\hline European Journal of Cardiovascular Nursing & 6 & European Journal of Cardiovascular Nursing & 2 \\
\hline International Nursing Review & 6 & Australian Journal of Rural Health & 1 \\
\hline Intensive and Critical Care Nursing & 4 & International Journal of Nursing Knowledge & 1 \\
\hline Journal of Psychiatric and Mental Health Nursing & 4 & International Journal of Nursing Studies & 1 \\
\hline Australian Journal of Rural Health & 3 & Journal of Obstetric Gynecologic and Neonatal Nursing & 1 \\
\hline Geriatric Nursing & 2 & Journal of Nursing Education & 1 \\
\hline International Journal of Mental Health Nursing & 2 & Journal of Nursing Management & 1 \\
\hline Journal for Nurse Practitioners & 2 & Journal of Nursing Research & 1 \\
\hline Journal of Obstetric Gynecologic and Neonatal Nursing & 2 & Journal of Transcultural Nursing & 1 \\
\hline Journal of Continuing Education in Nursing & 2 & Midwifery & 1 \\
\hline Midwifery & 2 & Nurse Education in Practice & 1 \\
\hline Nephrology Nursing Journal & 2 & Nurse Education Today & 1 \\
\hline Nursing Economics & 2 & Seminars in Oncology Nursing & 1 \\
\hline
\end{tabular}

Lactation ( $\mathrm{n}=10)$, Heart Lung $(\mathrm{n}=8)$, and the Journal of Advanced Nursing $(\mathrm{n}=7)$. The most original articles and reviews were published in the International Nursing Review $(\mathrm{n}=4)$, the Journal of Clinical Nursing $(\mathrm{n}=3)$, and the Journal of Human Lactation $(\mathrm{n}=3)$.

Table 2 presents the most productive countries and institutions regarding COVID-19 papers. The most productive countries were the USA $(n=53)$ and Australia $(n=25)$, which together produced approximately $60 \%$ of all publications. England and China published 18 and 15 publications, respectively. The most original articles and reviews were published from the USA ( $(n=16)$, Australia $(n=6)$, and England $(n=4)$. The University of Technology Sydney ( $\mathrm{n}=14)$, Johns Hopkins University $(\mathrm{n}=9)$, and the State University System of Florida $(n=7)$ were the top institutions publishing COVID-19 research. In addition, the International Council of Nurses, Johns Hopkins University, the University of Connecticut, the University of Technology Sydney, the University of Texas System each published three original articles or review papers.
Fig. 1 describes the bibliographic information of the included publications. With regard to the month of publication, the proportion of early access papers was the highest $(n=51$, $40.8 \%$ ), followed by studies published in May to June ( $\mathrm{n}=43$, $34,4 \%)$, July to August $(\mathrm{n}=23,18.4 \%)$, and March to April $(n=8,6.4 \%)$. None of the studies were published before February 2020 . Editorial materials accounted for $60.0 \%$ of the articles $(n=75)$, followed by original articles $(n=27,21.6 \%)$, letters $(\mathrm{n}=19,15.2 \%)$, and reviews $(\mathrm{n}=4,3.2 \%)$. The studies were published most frequently in Q1 journals $(\mathrm{n}=73$, $58.4 \%)$, followed by Q2 $(\mathrm{n}=25,20.0 \%), \mathrm{Q} 3(\mathrm{n}=14,11.2 \%)$, and Q4 ( $\mathrm{n}=13,10.4 \%)$ journals. Table 3 shows differences in the types of publications and the quartiles of the journals according to the month of publication. There was no significant difference in the type of publication according to the month of publication $\left(\chi^{2}=11.48, P=0.244\right)$. However, a statistically significant difference in the IF quartile of the journals was found according to the month of publication $\left(\chi^{2}=56.34\right.$, $\mathrm{P}<0.001)$, indicating that a disproportionately high propor- 
Table 2. The most productive countries and institutions regarding COVID-19 publications in nursing journals

\begin{tabular}{|c|c|c|c|c|}
\hline & Country & Number & Institution & Number \\
\hline \multirow[t]{19}{*}{ Overall } & USA & 53 & University of Technology Sydney & 14 \\
\hline & Australia & 25 & Johns Hopkins University & 9 \\
\hline & England & 18 & State University System of Florida & 7 \\
\hline & China & 15 & University of Connecticut & 5 \\
\hline & Italy & 8 & University of Texas System & 5 \\
\hline & Canada & 6 & Linkoping University & 4 \\
\hline & New Zealand & 6 & University of Birmingham & 4 \\
\hline & Netherlands & 5 & University of California System & 4 \\
\hline & Sweden & 5 & University of Florida & 4 \\
\hline & France & 4 & University of Hong Kong & 4 \\
\hline & Scotland & 4 & University of Pennsylvania & 4 \\
\hline & Switzerland & 4 & Utrecht University & 4 \\
\hline & Denmark & 3 & Utrecht University Medical Center & 4 \\
\hline & Spain & 3 & Yale University & 4 \\
\hline & Thailand & 3 & & \\
\hline & Brazil & 2 & & \\
\hline & Ireland & 2 & & \\
\hline & Japan & 2 & & \\
\hline & Taiwan & 2 & & \\
\hline \multirow[t]{9}{*}{ Original article + review } & USA & 16 & The International Council of Nurses & 3 \\
\hline & Australia & 6 & Johns Hopkins University & 3 \\
\hline & England & 4 & University of Connecticut & 3 \\
\hline & Canada & 3 & University of Technology Sydney & 3 \\
\hline & Switzerland & 3 & University of Texas System & 3 \\
\hline & Denmark & 2 & Connecticut Children S Medical Center & 2 \\
\hline & France & 2 & State University System of Florida & 2 \\
\hline & China & 2 & University of Florida & 2 \\
\hline & Scotland & 2 & University of Texas El Paso & 2 \\
\hline
\end{tabular}
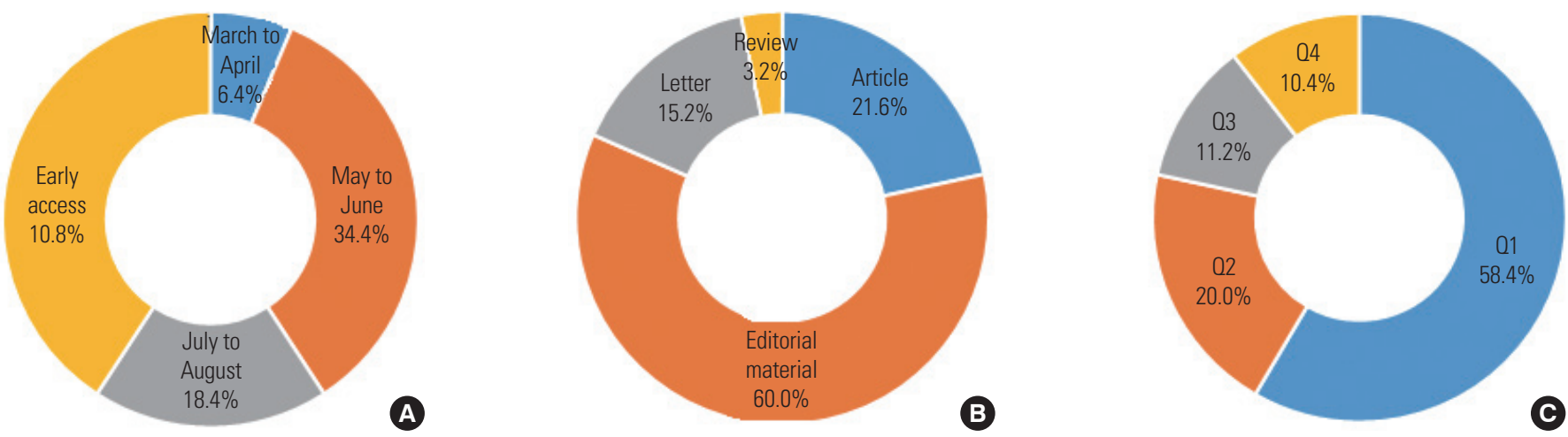

Fig. 1. Bibliographic information of publications according to (A) publication month, (B) type of publication, and (C) journal ranking. 
Table 3. Bibliographic information according to publication date

\begin{tabular}{|c|c|c|c|c|c|c|c|}
\hline & & March to April & May to June & July to August & Early access & $X^{2}$ & P-value \\
\hline \multirow{3}{*}{ Type } & Editorial & $3(37.50)$ & 30 (69.77) & $13(56.52)$ & $29(56.86)$ & & \\
\hline & Letter & $1(12.50)$ & $2(4.65)$ & $6(26.09)$ & $10(19.61)$ & & \\
\hline & Review & $1(12.50)$ & $1(2.33)$ & $1(4.35)$ & $1(1.96)$ & & \\
\hline \multirow{2}{*}{ Quartile } & 03 & $0(0.00)$ & $11(25.58)$ & $0(0.00)$ & $3(5.88)$ & & \\
\hline & 04 & $3(37.50)$ & $9(20.93)$ & $0(0.00)$ & $1(1.96)$ & & \\
\hline
\end{tabular}

Values are presented as number (\%).

Table 4. Topics of the 10 data-based original articles or reviews of nursing articles related to COVID-19

\begin{tabular}{llll}
\hline Document type & \multicolumn{1}{c}{ Topic } & \multicolumn{1}{c}{ Category } & DOI \\
\hline Article & Health care training app for COVID-19 patients & Nurse training & $10.1016 / j . n e d t .2020 .104479$ \\
Article & Research methodology of nursing education & Research methodology & $10.3928 / 01484834-20200422-02$ \\
Article & Nurse's willingness to practice in an epidemic area in China & Psychosocial status of nurses & $10.1111 /$ jan.14434 \\
Article & Nursing terminology & Nurse training & $10.1111 / 2047-3095.12291$ \\
Article & Work stress of Chinese nurses & Psychosocial status of nurses & $10.1111 /$ jonm.13014 \\
Review & Care for women and infants, resources & Guideline & $10.1016 / j . j 0 g n .2020 .04 .001$ \\
Review & Clinical practice, public health Intervention & Guideline & $10.1177 / 1043659620917724$ \\
Review & Recommendations for the patients, management program & Guideline & $10.1177 / 1474515120934057$ \\
Review & Remote health care for cardiovascular patients & Guideline & $10.1177 / 1474515120924530$ \\
Systemic review & Mask efficiency & Nurse training & $10.1016 / j . j \mathrm{jn}$.rstu.2020.103629 \\
\hline
\end{tabular}

tion of early access papers were published in Q1 journals.

Content analysis of the 31 reviews and articles showed that there were ten data-based original articles or reviews including systematic reviews (Dataset 1). The topics of the original articles were as follows: development of a health care training app for COVID-19 patients, research methodology in nursing education, nurses' willingness to practice in an epidemic area in China, nursing terminology related to COVID-19, and work stress among Chinese nurses. One systematic review dealt with the efficacy of masks for preventing COVID-19 infection (Table 4).

\section{Discussion}

Interpretation: In the present study, which focused on papers published in nursing journals, we aimed to provide information on the current status of publications related to the COVID-19 pandemic. A total of 125 studies published in nursing journals were found in the Web of Science database, and we analyzed the related bibliometric data. Within only half a year, a large number of papers related to COVID-19 have appeared in nursing journals. The most productive country was the USA, which is in line with the results of other bibliometric studies of scientific literature related to COVID-19 [7]. This could be explained by the fact that the USA is one of the most prolific countries in regard to nursing research [11] and currently has the highest number of confirmed COVID-19 cases [4]. Researchers from East Asian countries, including China, where epidemic peaked in early 2020, published the highest number of papers on COVID-19 in medical journals [7,8], but the number of studies from these countries published in nursing journals was 15 , accounting for a relatively small proportion of the documents in our findings.

Interestingly, over $50 \%$ of the examined publications were editorial materials, which suggests that many nursing journals have shown a great interest in COVID-19. However, a relatively low proportion (20.5\%) of original articles and only four review papers regarding COVID-19 have been published 
in nursing journals, which is lower than the number of studies analyzing COVID-19 that have been published in other fields of science $[7,8]$. Generally, editorial materials that introduce the editor or editorial board to the specific issue at hand have less real scientific content [12] and a lower quality of evidence [13]. The small number of published original articles can be explained by the long publication process of nursing journals. A previous study that analyzed the publication efficiency of top nursing journals reported that the publication process takes approximately 2.5 to 3 years [14]. Although the time between data collection and manuscript submission accounts for $62.5 \%$ of that time, the period from manuscript submission to acceptance and online publication takes almost 12 months on average [14].

The highest percentage of papers were published as early access, and many papers have also been published in recent months. Since our data were extracted in July, the total number of articles published in July to August must be higher than the publication number reflected in our results. In terms of journal ranking, approximately $60 \%$ of the examined papers were published in Q1 journals. Interestingly, this tendency was more obvious in July to August and early access publications; for example, over $80 \%$ of the early-access literature and $60 \%$ of the July to August articles were published in Q1 journals, which might be explained by the fact that many researchers have submitted manuscripts to more highly ranked journals. Another explanation for this outcome is the possibility that some highly ranked journals might have shortened their publication process, but further research will be needed to understand these possible factors. Therefore, addressing topics of interest to readers, such as COVID-19 (e.g., by releasing special issues), is needed to increase a journal's impact level and readership. This approach could also contribute to raising the citation index, since many nursing studies on $\mathrm{CO}$ VID-19 are likely to be published during the COVID-19 pandemic and even after the virus is eradicated.

The content analysis showed that there were 10 data-based original articles or reviews based on data collected by a survey or literature search. In Web of Science, the document type "article" comprised a variety of publication types. Therefore, if the category of original articles is limited to studies derived from experimental data or literature reviews, the number of original articles is reduced. The reviews mostly dealt with guidelines for nursing care, while the original articles focused on the training of nurses and their psychosocial status. Therefore, it is still too early for nursing researchers to publish articles on empirical research related to COVID-19.

Limitation: This study has several limitations. First, Science Citation Index Expanded and Social Sciences Citation Index do not index all nursing journals, and we did not include journal articles that were included in other databases, such as Scopus. Another limitation is the inclusion of only Englishlanguage articles in the study. For example, the Chinese literature might have been underestimated, and as China was found to have published the highest number of papers in previous studies $[4,9]$. Second, citation data were not examined in this study because most of the publications have not been cited in other studies, most likely because not enough time has elapsed for such articles to be cited. Last, the latest articles published after the middle of July 2020 were not included in this analysis. As of July 2020, the COVID-19 pandemic is not over yet; therefore, future bibliometric analyses will be needed, including citation data and a more comprehensive examination of nursing studies from several databases.

Conclusion: By demonstrating the current status of the literature through an analysis of publications found in nursing journals, this study provides meaningful information for nursing researchers and editorial board members. In the COVID-19 era, the timely accumulation of knowledge can be used to make improvements related to the health and health care of not only people affected with COVID-19, but all humankind. Journal articles are recognized as the main method for knowledge diffusion. Thus, it is necessary to encourage nurses to contribute to research on COVID-19 and to integrate that knowledge into nursing practice.

\section{Conflict of Interest}

No potential conflict of interest relevant to this article was reported.

\section{Data Availability}

Dataset file is available from: the Harvard Dataverse at: https:// doi.org/10.7910/DVN/6CZCHI

Dataset 1. Raw data from the Web of Science website

\section{References}

1. Than HM. Essential case management practices amidst the novel coronavirus disease 2019 (COVID-19) crisis: part 1: tele-case management, surge capacity, discharge planning, and transitions of care. Prof Case Manag 2020;25:248-66. https://doi.org/10.1097/NCM.0000000000000454

2. World Health Organization. WHO Director-General's opening remarks at the media briefing on COVID-19: 11 March 2020 [Internet]. Geneva: World Health Organization; 2020 [cited 2020 Jul 17]. Available from: https://www.who.int/dg/ speeches/detail/who-director-general-s-opening-remarks-at- 
the-media-briefing-on-covid-19---11-march-2020

3. World Health Organization. WHO coronavirus disease (COVID-19) dashboard [Internet]. Geneva: World Health Organization; 2020 [cited 2020 Jul 17]. Available from: https://covid19.who.int/

4. Chahrour M, Assi S, Bejjani M, et al. A bibliometric analysis of COVID-19 research activity: a call for increased output. Cureus 2020;12:e7357. https://doi.org/10.7759/cureus.7357

5. Daly J, Jackson D, Anders R, Davidson PM. Who speaks for nursing? COVID-19 highlighting gaps in leadership. J Clin Nurs 2020;29:2751-2. https://doi.org/10.1111/jocn.15305

6. Jiang L, Broome ME, Ning CY. The performance and professionalism of nurses in the fight against the new outbreak of COVID-19 epidemic is laudable. Int J Nurs Stud 2020;107: 103578. https://doi.org/10.1016/j.ijnurstu.2020.103578

7. Haghani M, Bliemer MC, Goerlandt F, Li J. The scientific literature on coronaviruses, COVID-19 and its associated safety-related research dimensions: A scientometric analysis and scoping review. Saf Sci 2020;129:104806. https:// doi.org/10.1016/j.ssci.2020.104806

8. Lou J, Tian SJ, Niu SM, et al. Coronavirus disease 2019: a bibliometric analysis and review. Eur Rev Med Pharmacol Sci 2020;24:
3411-21. https://doi.org/10.26355/eurrev_202003_20712

9. Hamidah I, Sriyono S, Hudha MN. A bibliometric analysis of Covid-19 research using VOSviewer. Indones J Sci Technol 2020;5:209-16. https://doi.org/10.17509/ijost.v5i2.24522

10. Dehghanbanadaki H, Seif F, Vahidi Y, et al. Bibliometric analysis of global scientific research on coronavirus (COVID-19). Med J Islam Repub Iran 2020;34:354-62. https:// doi.org/10.34171/mjiri.34.51

11. Gimenez-Espert MD, Prado-Gasco VJ. Bibliometric analysis of six nursing journals from the Web of Science, 2012-2017. J Adv Nurs 2019;75:543-54. https://doi.org/10.1111/jan.13868

12. van Leeuwen T, Costas R, Calero-Medina C, Visser M. The role of editorial material in bibliometric research performance assessments. Scientometrics 2013;95:817-28. https:// doi.org/10.1007/s11192-012-0904-5

13. Burns PB, Rohrich RJ, Chung KC. The levels of evidence and their role in evidence-based medicine. Plast Reconstr Surg 2011;128:305-10. https://doi.org/10.1097/PRS.0b013e318219c171

14. Palese A, Coletti S, Dante A. Publication efficiency among the higher impact factor nursing journals in 2009: a retrospective analysis. Int J Nurs Stud 2013;50:543-51. https:// doi.org/10.1016/j.ijnurstu.2012.08.019 